\title{
Insights into the role of extracellular polysaccharides in Burkholderia adaptation to different environments
}

\author{
Ana S. Ferreira ${ }^{1}$, Inês N. Silva ${ }^{1}$, Vítor H. Oliveira ${ }^{1}$, Raquel Cunha ${ }^{1}$ and Leonilde M. Moreira ${ }^{1,2}$ * \\ ${ }^{1}$ Institute for Biotechnology and Bioengineering, Centre for Biological and Chemical Engineering, Instituto Superior Técnico, Lisboa, Portugal \\ ${ }^{2}$ Department of Bioengineering, Instituto Superior Técnico, Lisboa, Portugal
}

\author{
Edited by: \\ Joanna Goldberg, University of \\ Virginia Health System, USA \\ Reviewed by: \\ Tonyia Eaves-Pyles, University of \\ Texas Medical Branch, USA \\ Miguel A. Valvano, University of \\ Western Ontario, Canada \\ Roberto Rizzo, University of Trieste, \\ Italy \\ ${ }^{*}$ Correspondence: \\ Leonilde M. Moreira, Instituto \\ Superior Técnico, Torre Sul, Piso 6, \\ Avenida Rovisco Pais, 1049-001 \\ Lisboa, Portugal. \\ e-mail:Imoreira@ist.utl.pt
}

The genus Burkholderia comprises more than 60 species able to adapt to a wide range of environments such as soil and water, and also colonize and infect plants and animals. They have large genomes with multiple replicons and high gene number, allowing these bacteria to thrive in very different niches. Among the properties of bacteria from the genus Burkholderia is the ability to produce several types of exopolysaccharides (EPSs). The most common one, cepacian, is produced by the majority of the strains examined irrespective of whether or not they belong to the Burkholderia cepacia complex (BcC). Cepacian biosynthesis proceeds by aWzy-dependent mechanism, and some of the B. cepacia exopolysaccharide (Bce) proteins have been functionally characterized. In vitro studies showed that cepacian protects bacterial cells challenged with external stresses. Regarding virulence, bacterial cells with the ability to produce EPS are more virulent in several animal models of infection than their isogenic non-producing mutants. Although the production of EPS within the lungs of cystic fibrosis (CF) patients has not been demonstrated, the in vitro assessment of the mucoid phenotype in serial Bcc isolates from CF patients colonized for several years showed that mucoid to non-mucoid transitions are relatively frequent. This morphotype variation can be induced under laboratory conditions by exposing cells to stress such as high antibiotic concentration. Clonal isolates where mucoid to non-mucoid transition had occurred showed that during lung infection, genomic rearrangements, and mutations had taken place. Other phenotypic changes include variations in motility, chemotaxis, biofilm formation, bacterial survival rate under nutrient starvation and virulence. In this review, we summarize major findings related to EPS biosynthesis by Burkholderia and the implications in broader regulatory mechanisms important for cell adaptation to the different niches colonized by these bacteria.

Keywords: exopolysaccharide, cepacian, biosynthesis, Burkholderia, cystic fibrosis, mucoid variation, virulence, persistence

\section{INTRODUCTION}

Extracellular polysaccharides or exopolysaccharides (EPSs) are high-molecular weight sugar-based polymers that are synthesized and secreted by many microorganisms. The importance of their production has been studied in many bacteria. Typically EPSs play a role in bacterial adaptation to different stress conditions, being involved in the establishment of symbiotic and pathogenic relationships with hosts and being suggested to be a major component of biofilm mature structures. The genus Burkholderia also has the ability to produce EPS and at least seven different exopolysaccharides have been identified and their structure determined (Table 1). Some strains produce a single exopolysaccharide while others produce mixtures. For example, Burkholderia cepacia IST408 produces cepacian only (Cescutti et al., 2000), but Burkholderia cenocepacia C9343 produces simultaneously PS-I, cepacian (PS-II), and $\alpha$-1,6-glucan (Conway et al., 2004). The most common EPS produced by Burkholderia is cepacian and has been identified in different species, including environmental isolates associated with plants and in both clinical and environmental isolates from B. cepacia complex (Bcc; Ferreira et al., 2010; Hallack et al., 2010). Furthermore, genes encoding proteins involved in cepacian synthesis are well conserved among Burkholderia, suggesting that cepacian is a common feature among the genus (Ferreira et al., 2010). Cepacian is composed of a branched acetylated heptasaccharide repeat-unit with $\mathrm{D}$ glucose, D-rhamnose, D-mannose, D-galactose, and D-glucuronic acid in the ratio of 1:1:1:3:1 (Cerantola et al., 1999; Cescutti et al., 2000).

Cepacian production depends on external factors. For instance, many strains unable to produce exopolysaccharide on LB medium start to over-produce it in rich glucose- or mannitol-based medium, such as S, SM, or YEM media (Richau et al., 2000a; Bartholdson et al., 2008; Zlosnik et al., 2008). Also, the type of EPS produced depends on external growth conditions as demonstrated for the production of EPS A and EPS B by Burkholderia kururiensis (Table 1; Hallack et al., 2010). These observations indicate that EPS production by Burkholderia is tightly regulated as a response to external conditions, which can be crucial in bacterial colonization 
Table 1 | Structural formula of the exopolysaccharide repeated units described in the literature.

\begin{tabular}{|c|c|c|c|}
\hline EPS/species & Structure & Observations & Reference \\
\hline $\begin{array}{l}\text { Cepacian (PS-II)/Bcc } \\
\text { and non-Bcc }\end{array}$ & 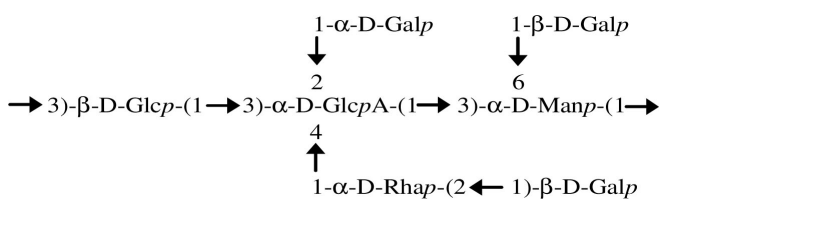 & $\begin{array}{l}\text { Environmental and clinical } \\
\text { isolates; bce-l and bce-ll genes } \\
\text { present in most Burkholderia } \\
\text { species; number of acetyl } \\
\text { groups is strain dependent } \\
\text { (two to four per RU) }\end{array}$ & $\begin{array}{l}\text { Cerantola et al. (1999), } \\
\text { Cescutti et al. (2000), } \\
\text { Hallack et al. (2010) }\end{array}$ \\
\hline PS-I/B. cepacia & $\rightarrow 3)-\beta-D-G l c p-(1 \rightarrow 3)-[4,6-O-(1$-carboxyethylidene $)]-\alpha-D-G a l p-(1 \rightarrow$ & Clinical isolate & Cerantola et al. (1996) \\
\hline Levan/B. cepacia & $\rightarrow 6)-\beta-D-F r u f-(2 \rightarrow$ & $\begin{array}{l}\text { This strain produces an EPS } \\
\text { mixture that includes levan }\end{array}$ & Cescutti et al. (2003) \\
\hline EPS A/B. kururiensis & 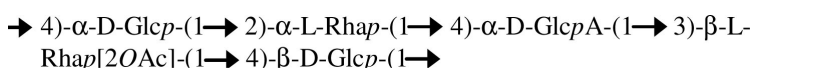 & $\begin{array}{l}\text { Endophytic diazotrophic } \\
\text { species }\end{array}$ & Mattos et al. (2001) \\
\hline EPS B/B. kururiensis & $\rightarrow 3$ - $\beta$-D-Glc $p-(1 \rightarrow 3)-\alpha-\mathrm{D}-\mathrm{Glc} p \mathrm{~A}-(1 \rightarrow 3)-\alpha-\mathrm{D}-\mathrm{Man} p-(1 \rightarrow$ & $\begin{array}{l}\text { EPS B composed of a mixture } \\
\text { of two polymers: cepacian and } \\
\text { the octasaccharide polymer, } \\
\text { differing by the presence of a } \\
\text { terminal glycosyl residue }\end{array}$ & Hallack et al. (2010) \\
\hline $\begin{array}{l}\text { EPS/B. pseudomallei, } \\
\text { B. cepacia }\end{array}$ & $\begin{array}{l}\text { 5)- } \beta \text {-D-Kdop- }(2 \rightarrow 3)-\beta-\mathrm{D}-\mathrm{Gal} p 2 O \mathrm{Ac}-(1 \rightarrow 4)-\alpha-\mathrm{D}-\mathrm{Gal} p-(1 \rightarrow 3)-\beta-\mathrm{D}- \\
\text { Gal } p-(1 \rightarrow\end{array}$ & One acetyl group per RU & $\begin{array}{l}\text { Nimtz et al. (1997), } \\
\text { Cescutti et al. (2003) }\end{array}$ \\
\hline EPS/B. caribensis & 3)- $\beta$-D-Glc $p-(1 \rightarrow 4)-\beta-$ D-Glc $p-(1 \rightarrow 4)-\alpha-$ L-dTal $p-(1 \rightarrow$ & $\begin{array}{l}\text { Isolated from rhizosphere and } \\
\text { important for soil aggregation }\end{array}$ & $\begin{array}{l}\text { Vanhaverbeke et al. } \\
\text { (2001) }\end{array}$ \\
\hline
\end{tabular}

Main species from where different EPS were identified and relevant information associated with strain source or EPS characteristics is provided. Gal, galactose; Glc, glucose; GlcA, glucuronic acid; Man, mannose; Rha, rhamnose; Fru, fructose; Kdo, 3-deoxy-D-manno-2-octulosonic acid; 6dTal, 6-deoxy-talose; OAc, acetyl substituent; $R U$, repeat-unit.

and adaptation to different environments and hosts. Since cepacian is the major EPS produced by Burkholderia, this review will focus on the present knowledge concerning cepacian biosynthesis, regulation, and possible importance in bacteria lifestyle.

\section{GENES AND PROTEINS INVOLVED IN CEPACIAN BIOSYNTHESIS}

Genes involved in cepacian biosynthesis are located within bce-I and bce-II gene clusters (Figure 1A; Moreira et al., 2003; Ferreira et al., 2010). Comparative genomic studies indicate that the two clusters are present in all sequenced Burkholderia genomes, with the exception of the intracellular endosymbiotic Burkholderia rhizoxynica HKI 454 with both gene clusters absent and Burkholderia mallei having only bce-II. Isolates from these two species suffered genomic size reduction and loss of many genes as indicated by the 3.6-Mbp of B. rhizoxynica genome and the 5.2- to 5.9-Mbp of $B$. mallei genomes in comparison with the 6.3- to 9.7-Mbp of the genomes from the remaining Burkholderia species. Depending on the species, the two gene clusters can be located together in the same genomic region or separated by hundreds of kilobase pairs (Ferreira et al., 2010). The phylogenetic tree based on the concatenation of all Bce protein sequences showed three distinct groups (Figure 1B). Group I includes clinical and environmental isolates from the Burkholderia cepacia complex, all of them having the two bce clusters located apart from each other. Group II contains animal and plant pathogenic non- $B c c$ isolates also having the two clusters in different genomic locations. Group III harbors non-pathogenic rhizosphere and plant-associated strains 


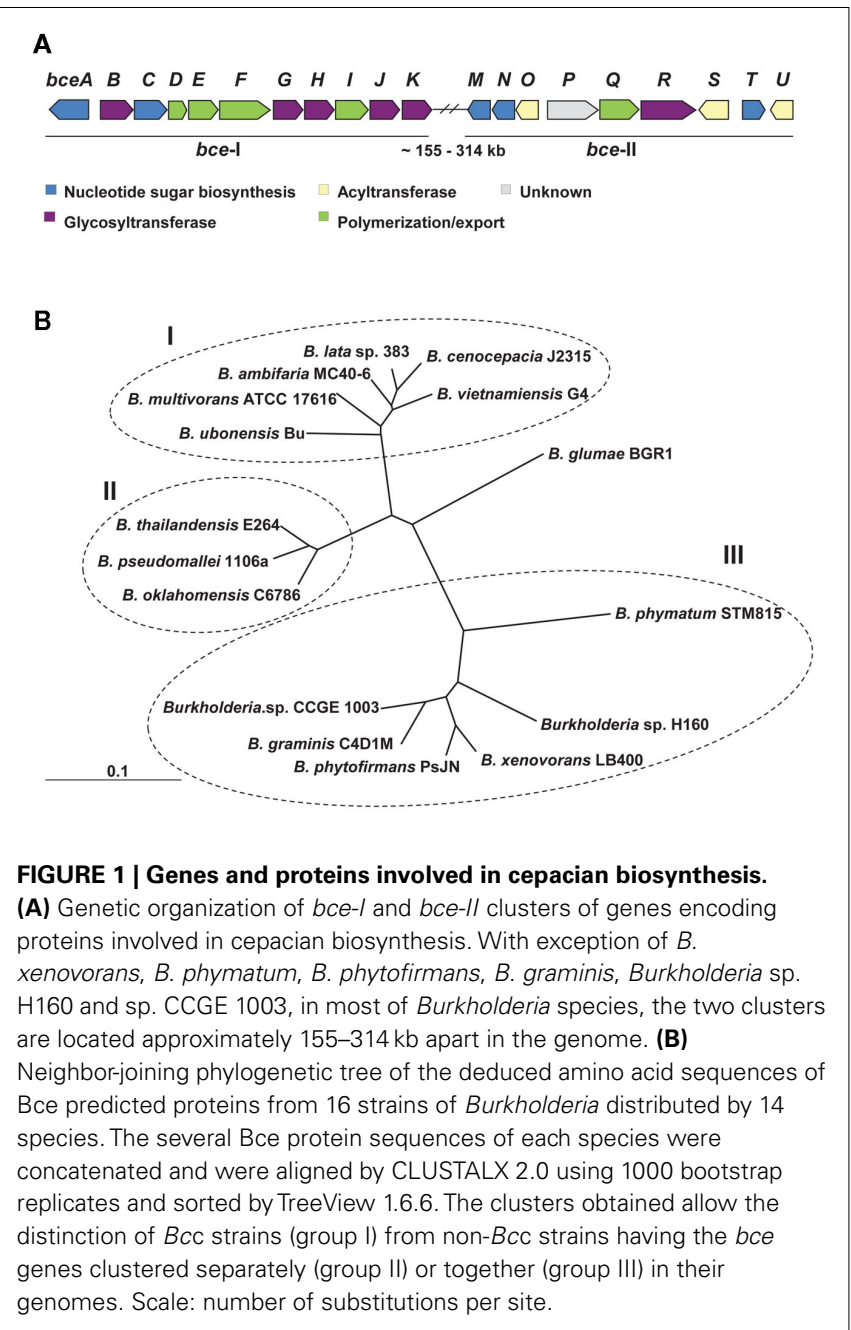

having $b c e-I$ and $b c e-I I$ gene clusters together. The rice pathogen Burkholderia glumae BGR1, evolutionarily close to groups I and II strains, also has the bce gene clusters into different genomic locations.

Cepacian biosynthesis starts with the formation of activated sugar-nucleotide precursors required for the synthesis of the repeat-unit building blocks (Figure 2A; Richau et al., 2000b). Several of the enzymes involved in the process are encoded within the $b c e-I$ and $b c e-I I$ gene clusters. Gene $b c e A$ encodes a bifunctional protein providing the first and third steps (phosphomannose isomerase and GDP-D-mannose pyrophosphorylase activities) for converting fructose-6-phosphate from central metabolism into GDP-D-mannose (Figure 2A; Sousa et al., 2007a, 2008). A bceA gene insertion mutant was still capable of producing half the amount of EPS of the parental strain $B$. cepacia IST408, showing that other enzymes producing GDP-Dmannose are present in this microorganism (Sousa et al., 2007a). Gene $b c e C$ encodes a UDP-glucose dehydrogenase responsible for the NAD-dependent twofold oxidation of UDP-D-glucose to UDP-D-glucuronic acid (Loutet et al., 2009). Its 3D-structure was recently determined and the tyrosine residue at position 10 was found to be the key catalytic residue in the final hydrolysis

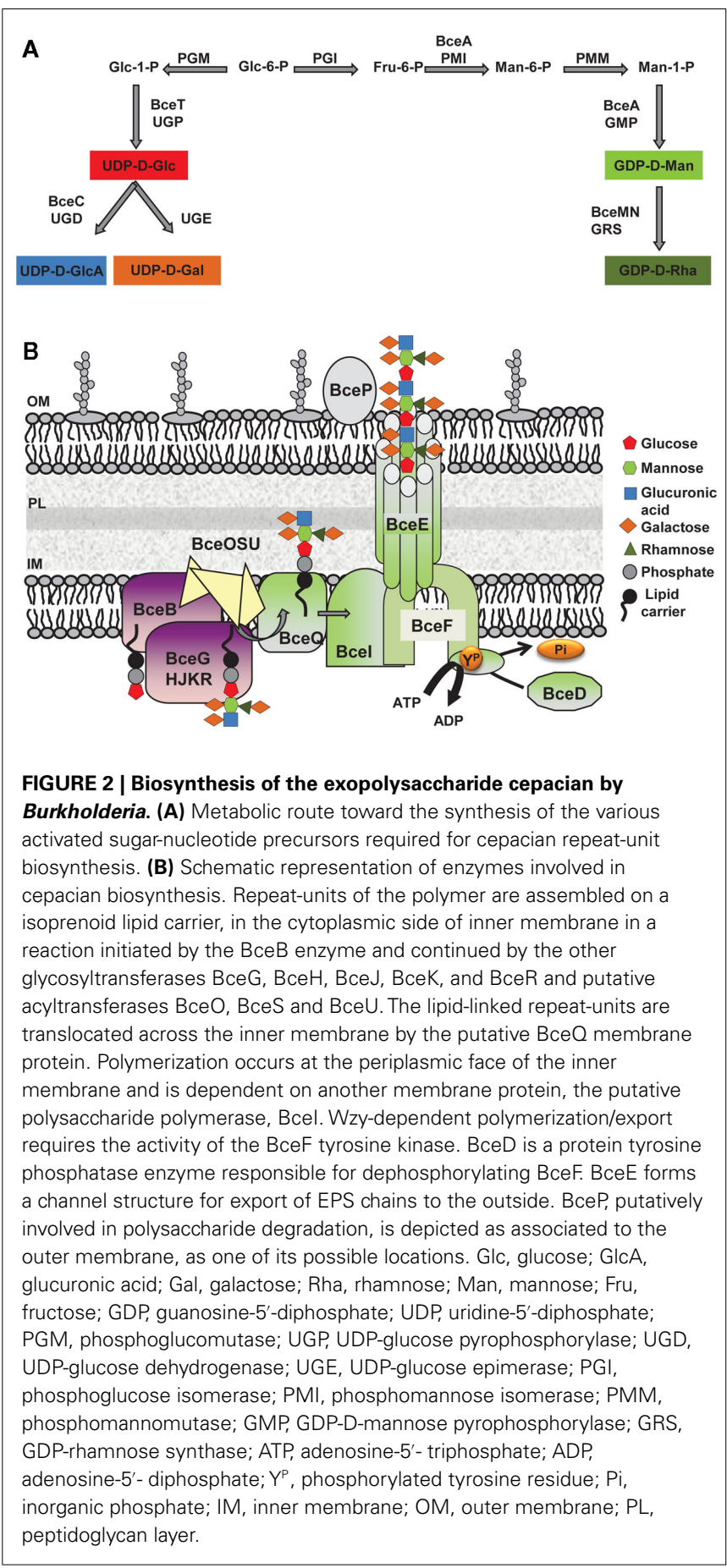

of the enzymatic thioester intermediate (Rocha et al., 2011). Although there is no experimental demonstration, bceT gene product must provide the activity of UDP-glucose pyrophosphorylase for the synthesis of UDP-D-glucose and bceN and $b c e M$ gene products provide the activities of GDP-mannose4,6-dehydratase (GMD) and a GDP-6-deoxy-D-lyxo-4-hexulose reductase (RMD) for GDP-D-rhamnose synthesis (Figure 2A; Ferreira et al., 2010). The genes encoding the enzymes UDP-glucose epimerase required for the formation of UDP-D-galactose and 
phosphoglucomutase (PGM), phosphoglucose isomerase (PGI), and phosphomannomutase (PMM) required for the synthesis of some of the intermediary precursors are absent from bce-I and $b c e-$ II gene clusters. Since the products of these enzymatic reactions are involved in other polysaccharide biosynthetic pathways, these enzyme-encoding genes are located elsewhere in the genome. In silico analysis using B. cenocepacia J2315 genome sequence shows that BCAL3113 encodes an enzyme with the putative function of PGM and PMM; BCAL1990 encodes the putative PGI enzyme; and BCAL3117 encodes a putative UDP-glucose epimerase.

The synthesis of the activated sugar-nucleotide precursors is followed by the assembly of the heptasaccharide repeat-unit, catalyzed by glycosyltransferase proteins encoded by $b c e B, b c e G, b c e H$, $b c e J, b c e K$, and bceR genes (Figure 2B). BceB is an inner membrane protein that catalyzes the transfer of glucose-1-phosphate to the isoprenoid lipid carrier (Videira et al., 2005). The specific sugar added by the remaining Bce glycosyltransferases has not yet been identified. BceG is predicted to belong to the CAZy GT-2 family of enzymes that transfer glycosyl residues by an inverting mechanism and adopt a GT-A fold having a single Rossmann-like domain for nucleotide binding (Coutinho et al., 2003). BceH, BceJ, and BceK are predicted to belong to CAZy GT-1 family, which comprises retaining glycosyltransferases that adopt a GT-B fold characteristic of proteins that have two Rossmann-like domains. The protein encoded by $b c e R$ gene is predicted to be a bifunctional enzyme with two glycosyltransferase domains of CAZy GT-4 family with a retaining mechanism of glycosyl residue transfer and adopting a GT-B fold. BceR has been shown to be essential for cepacian biosynthesis, as confirmed by the absence of exopolysaccharide in the growth medium of $B$. cepacia IST408 bceR gene insertion mutant (Ferreira et al., 2010). BceG, BceH, and BceR are probably cytoplasmic proteins, whereas $\mathrm{BceJ}$ and $\mathrm{BceK}$ are likely to possess a transmembrane domain, being integral membrane proteins. The order of sugar addition to the repeat-unit has been proposed (Cescutti et al., 2010) with the glucuronic acid being added to a trisaccharide composed of glucose and mannose substituted with galactose; then a second galactose is added to the glucuronic acid as the first substituent, followed by a rhamnose and galactose, as a second disaccharide substituent (Cescutti et al., 2010). Cepacian acetylation is predicted to occur during the repeat-unit assembly process, but the number of substitutions is strain dependent (Cescutti et al., 2010). The enzymes required for these modifications are putatively encoded by $b c e S$, $b c e T$, and $b c e U$ genes and are predicted to be located in the inner membrane. BceO and BceU exhibit nine predicted transmembrane domains, while BceS exhibits eight predicted transmembrane domains (Figure 2B; Ferreira et al., 2010). Despite a weak conservation at the amino acid level, $\mathrm{BceO}, \mathrm{BceS}$, and $\mathrm{BceU}$ are homologous to a series of proteins that define a family of membrane proteins involved in the acylation of carbohydrate moieties of extracytoplasmic molecules. An insertion mutant on bceS gene showed a reduction of the acetylation content of the EPS produced by B. multivorans ATCC 17616 by approximately $20 \%$, which confirms BceS involvement in the repeat-unit modification (Ferreira et al., 2010).
The steps after repeat-unit assembly are polymerization and export of the exopolysaccharide to the cell's surrounding. Available data indicates the assembly proceeds via the so-called Wzydependent pathway (Figure 2B). According to this model, the lipid carrier-linked heptasaccharide repeat-units are exported across the inner membrane by a flippase protein and polymerized at the periplasmic face of the inner membrane by a polysaccharide polymerase. Since the proteins encoded by bceQ and bceI genes, have 12 and 10 transmembrane domains, respectively, and show similarity with other Wzx flippases and Wzy polysaccharide polymerases, we postulated that BceI is the putative polymerase and BceQ the putative flippase. Both genes are essential for cepacian biosynthesis, as demonstrated by the EPS deficient phenotype of the B. cepacia IST408 insertion mutants for bceI and bceQ genes (Moreira et al., 2003; Ferreira et al., 2010). The proteins encoded by $b c e D$ and $b c e F$ genes are also involved in the EPS polymerization and export process (Figure $2 \mathrm{~B}$ ). BceF belongs to the bacterial tyrosine kinase (BY-kinase) family which includes Wzc homologs predicted to act as the polysaccharide copolymerase component (Whitfield, 2006). BceF possesses two transmembrane domains that flank a large periplasmic domain and a cytoplasmic located C-terminal region. The periplasmic domain is predicted to adopt a coiled-coil structure important for interaction with other protein (such as BceE). Tocilj et al. (2008) propose that this periplasmic domain is the one affecting polysaccharide chain length, and that by interacting with the export component facilitates transfer of the polymer through the periplasm. The C-terminal cytoplasmic domain of BceF has the conserved Walker A and Walker B ATP-binding motifs and a tyrosine-rich cluster (Moreira et al., 2003; Ferreira et al., 2007). Site-directed mutagenesis was used to confirm the importance of the Walker A motif on BceF tyrosine autophosphorylation activity (Ferreira et al., 2007), but the role of tyrosine phosphorylation in cepacian biosynthesis is unknown. BceD is a phosphotyrosine phosphatase (PTP) protein that interacts with BceF, promoting the BY-kinase tyrosine dephosphorylation (Ferreira et al., 2007). BceE is a putative Wza homolog and therefore is predicted to be an outer membrane polysaccharide export protein (OPX) responsible for the final stage of polysaccharide export. OPX family of proteins are predicted to be lipoproteins that adopt an octameric configuration with a large central cavity that facilitates polysaccharide export through the periplasm and across the outer membrane (Dong et al., 2006). A B. cepacia IST408 bceE mutant is impaired in cepacian biosynthesis demonstrating the importance of this protein in the biosynthesis of this polymer (our unpublished data). Finally, bce-II gene cluster contains the bceP gene, encoding a protein of unknown function. BceP secondary structure was predicted to be exclusively composed of $\beta$-strands, and its location is unlikely the cytoplasm. It can be the periplasm, the outer membrane, or even the extracellular milieu. Structural homology modeling suggests that $\mathrm{BceP}$ resembles polysaccharide degrading enzymes such as Clostridium thermocellum xyloglucanase (PDB entry: $2 \mathrm{cn} 2$ ). Thus, BceP may be responsible for processing the polysaccharide before and/or after export depending on its cellular localization. 


\section{REGULATION OF CEPACIAN BIOSYNTHESIS}

Although the $b c e$-I and $b c e$-II gene clusters encode most of the structural proteins required for cepacian biosynthesis, the genes directly involved in the regulation of EPS production have not been identified. While $\operatorname{alg} U$ and mucA genes encode a transcription factor and anti-sigma factor, respectively, responsible for the control of the alginate biosynthetic operon in Pseudomonas aeruginosa (Ramsey and Wozniak, 2005), the B. cepacia AlgU homolog (RpoE) is not required for exopolysaccharide biosynthesis as demonstrated for a rpoE knockout mutant (Devescovi and Venturi, 2006). Although the mechanisms/players involved in the regulation of cepacian biosynthesis are unclear, there are evidences of the involvement of quorum sensing (QS) regulation at transcriptional level and protein tyrosine phosphorylation/dephosphorylation at post-translational level.

Recent studies on the plant-associated B. kururiensis M130, B. xenovorans LB400, and B. uname MTI-641 indicate that the $N$-acyl homoserine lactones (AHL) synthase BraI and the QS transcriptional regulator BraR are involved in the regulation of EPS biosynthesis. The mutants obtained for braI and $b r a R$ genes were less mucoid and the total sugar content of the growth culture decreased considerably (Suarez-Moreno et al., 2010). This phenotype could be reversed by external supplementation of 3-oxo-C14-HSL or 3oxo-C12-HSL, depending on the species (Suarez-Moreno et al., 2010). Such results confirm that QS positively controls EPS production in these plant-associated species, still the targets of such regulation cascade are unknown. Furthermore, the BraI/R system is not conserved in the Burkholderia genus and 3-oxo-AHLs are not produced by many species. For instance, in Bcc bacteria, the most well conserved QS system consists of CepI and CepR which produces and responds to C6-HSL and C8-HSL (Sokol et al., 2003). Whether CepI/CepR system mediated QS is able to regulate EPS production in the different $B c c$ species still needs confirmation.

Another mechanism that may be involved in regulating cepacian biosynthesis at the transcriptional level is mediated by the RNA chaperone Hfq. This protein is involved in the regulation of target mRNAs by small regulatory non-coding RNAs (Sousa et al., 2010). Deletion of $h f q$ gene of $B$. cepacia IST408 strongly reduces cepacian production. Whether a small RNA molecule is directly responsible for this phenotype or is a consequence of the pleiotropic effects caused by loss of this gene still has to be determined.

Regarding post-translational regulation, it is established that cycles of tyrosine phosphorylation and dephosphorylation, mediated by BY-kinases and PTP proteins, control the amount and the molecular weight of several bacterial exopolysaccharides (Vincent et al., 2000; Wugeditsch et al., 2001). However, the exact mechanism of such regulation is not clear. One hypothesis is that the BY-kinase tyrosine phosphorylation/dephosphorylation cycles interfere with the interaction with the outer membrane export OPX proteins by introducing conformational changes in the BY-kinase/OPX complex, perhaps affecting polymerization and/or export of the polysaccharide to the cell surrounding (Collins et al., 2006). Alternatively, BY-kinases may phosphorylate exogenous substrates, including proteins involved in sugar-nucleotide precursor's formation and repeat-unit assembly, thereby controlling their activity and consequently polysaccharide production. This was shown for colanic acid biosynthesis in E. coli in which the UDP-glucose dehydrogenase protein is phosphorylated by the Wzc BY-kinase, influencing the amount of EPS (Lacour et al., 2008). In Streptococcus thermophilus the EPS priming glycosyltransferase EpsE was shown to be regulated by the tyrosine kinase EpsD (Minic et al., 2007). Concerning cepacian biosynthesis, the disruption of the bceF gene abolishes cepacian production, while the bceD mutant shows a reduction of about $25 \%$ of cepacian production, and the EPS has a lower molecular weight than that produced by the parental strain (Ferreira et al., 2007). Therefore, BceF BYkinase and BceD PTP protein may be involved in the regulation of the amount and molecular weight of cepacian, even though there are no studies available regarding their exact role in polymerization/export or in regulating the activity of the enzymes involved in sugar-nucleotide precursors biosynthesis and repeat-unit assembly.

\section{ROLE OF BURKHOLDERIA EXOPOLYSACCHARIDES IN ADAPTATION TO DIFFERENT NICHES}

Bacterial extracellular polysaccharides have been described as pathogenicity determinants in humans, livestock, and plant infections; as important in the establishment of symbiotic interactions between bacteria and plants; and as a barrier to harmful compounds (Fraysse et al., 2003; Lebeer et al., 2011; Nielsen et al., 2011). Depending on the ecological niche, exopolysaccharides help bacteria to colonize different environments. Given that cepacian is ubiquitous in the genus Burkholderia, it would be expected that this EPS plays an important role in bacterial adaptation to different conditions including host/bacteria interactions. Figure 3 summarizes the current knowledge on the relevance of exopolysaccharides in Burkholderia biology. Interaction between plants and bacteria is often correlated to the ability of bacteria to produce EPSs, regardless being pathogenic or symbiotic. In pathogenic interactions, EPS contributes to the initial plant colonization and enhances bacteria survival within the plant host tissues during the course of infection, as described for Agrobacterium, Erwinia, and Pseudomonas (Denny, 1995). On the other hand, EPSs are important in the establishment of symbiotic interactions such as in biological nitrogen fixation symbiosis between rhizobia and leguminous plants

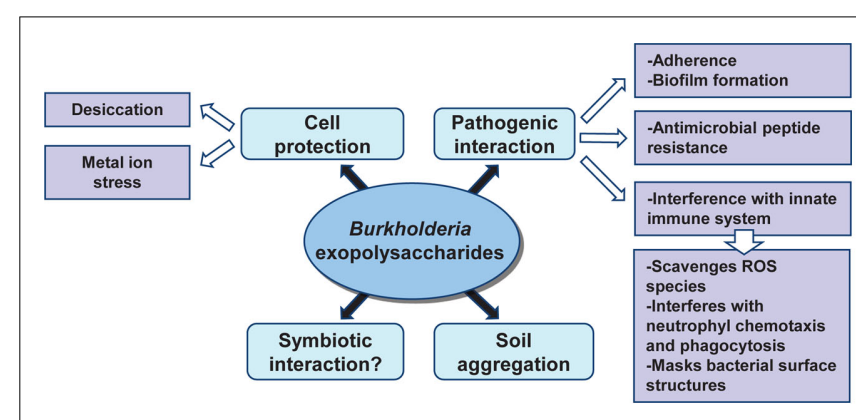

FIGURE 3 | Roles of Burkholderia exopolysaccharides in the adaptation to different niches. 
(Gonzalez et al., 1996). Since the first description of pathogenicity in the $B c c$ bacteria was based on sour skin rot of onion bulbs, Bartholdson et al. (2008) investigated EPS production in Bcc isolates and their onion-associated phenotype. Although the onion carbohydrates induced EPS production, no correlation between exopolysaccharide production ability of the tested strains and tissue onion maceration was found. Therefore, a possible role of the exopolysaccharides in interaction between Burkholderia and host plants has yet to be demonstrated. Nevertheless, the observation that the endophytic B. kururiensis exopolysaccharides can be modulated under growth conditions is potentially significant in terms of a possible endophytic-host plant interaction and further studies are needed (Hallack et al., 2010).

Since many exopolysaccharides are high-molecular weight polymers, they form a hydrated anionic matrix that surrounds the cell protecting bacteria against environmental stresses. Also, the hygroscopic properties of the EPS may reduce the rate of water loss from cells and provide bacteria with means to survive drying and desiccation (Potts, 1994). A study performed with $B$. xenovorans LB400 and B. multivorans ATCC 17616 isolates has shown that the external supplementation of cepacian enhanced their desiccation tolerance when compared to a condition where no exopolysaccharide was present (Ferreira et al., 2010). Cepacian also protects Burkholderia cells against metal ion stress, namely high concentration of $\mathrm{Fe}^{2+}$ and $\mathrm{Zn}^{2+}$ (Ferreira et al., 2010). The metal-binding properties of EPS might be due to the occurrence of carbonyl, carboxyl, and hydroxyl groups within the EPS matrix that can complex cations and scavenge metals (Potts, 1994). The ability of Burkholderia strains to withstand desiccation and metal ion stress in the presence of the cepacian is an indication that this EPS may play a role in survival, thus representing an advantage for bacteria to thrive in adverse environments.

Exopolysaccharides influence the stability of soil aggregates by interacting with clay particles contributing to maintain the mechanical and physical properties of the soil on which plant roots are grown. Vanhaverbeke et al. (2003) used the exopolysaccharide produced by Burkholderia caribensis MWAP71 to determine the interactions between the polysaccharide and the soil at the molecular level and one of the hypothesis raised is that the disorganized conformations of the exopolysaccharide connects several clay layers, resulting in a network composed by clays partially dispersed within an EPS matrix, which enhances the hydration properties of the whole soil.

Burkholderia exopolysaccharides, particularly cepacian, was postulated as a virulence determinant of $B c c$ species infecting the airways of cystic fibrosis (CF) patients and its importance in pathogenicity was assessed by several in vitro and in vivo studies. For instance, infection of a BALB/c mouse pulmonary infection model with two clonal isolates of $B$. cenocepacia obtained from a CF patient and displaying a different morphotype (mucoid vs. non-mucoid) showed that the mucoid isolate persisted longer in the lungs (Conway et al., 2004). Similarly, the infection of the gp91 9 hox-1- mouse with EPS-producing bacteria of the species $B$. cepacia showed that the EPS-producing strain B. cepacia IST408 caused higher mice mortality than the non-EPS producer isogenic bceF and bceI mutants (Sousa et al., 2007b). In vitro studies confirmed the ability of Burkholderia exopolysaccharides to interfere with the innate immune system by neutralizing reactive oxygen species (Bylund et al., 2006), inhibiting neutrophil chemotaxis and interfering with phagocytosis of bacteria by human neutrophils (Conway et al., 2004; Bylund et al., 2006). The interference by the EPS in phagocytosis could possibly be explained by the masking of bacterial surface antigens recognized by immune cells. Although these data suggest that exopolysaccharides enhance bacterial virulence, there is no experimental demonstration of $B c c$ bacteria producing it within the lungs. In addition, EPSs are not the only virulence factor in $\mathrm{Bcc}$ since the most hostile strains, responsible for major clinical outbreaks and associated to a worst clinical outcome that includes the development of cepacia syndrome, are non-mucoid (Govan et al., 1993). Supporting this, a recent surveillance study showed that patients infected with non-mucoid $B c c$ strains experience a more rapid lung function decline (Zlosnik et al., 2011). Still, most of the clinical isolates from different $B c c$ species, with the exception of B. cenocepacia, are able to produce exopolysaccharide (Zlosnik et al., 2008).

Another import role attributed to polysaccharides is the mediation of bacterial resistance against antimicrobial peptides produced by epithelial and phagocytic cells. A study involving the human antimicrobial peptides cathelicidin LL-37 and $\beta$-defensin $\mathrm{hBD}-3$ as well as peptides from other mammals demonstrated that the antibacterial activity of these different peptides was considerably decreased in the presence of polysaccharides produced by the lung pathogens P. aeruginosa, Klebsiella pneumoniae, and Bcc members (Benincasa et al., 2009). Production of bacterial polysaccharides in the lungs of CF patients could contribute to a decreased efficacy of the host defense response and the concomitant establishment of a persistent infection by these bacteria.

Exopolysaccharides, proteins, and DNA are the main constituents of the mature biofilm matrix contributing for example to the persistence of chronic $P$. aeruginosa lung infections in $\mathrm{CF}$ patients (Hentzer et al., 2001). Bacterial biofilms cause chronic infections due to their increased tolerance to antibiotics and resistance to the immune system phagocytic cells. As a consequence, chronic inflammation develops, being the major cause of the lung tissue damage in CF (Hoiby et al., 2010). Bcc bacteria were also shown to produce biofilms in abiotic surfaces and on welldifferentiated human epithelial cells (Schwab et al., 2002) and the production of biofilms associated to a significant increase of resistance against the host immune system and antibiotic treatment in Burkholderia (Caraher et al., 2007). Mutants constructed on bce genes have confirmed the importance of cepacian in the formation of mature biofilms. Mutants unable to produce cepacian, such as a $b c e F$ insertion mutant, or mutants that produce a lower molecular weight form, such as bceD insertion mutant, exhibited a much thinner biofilm when compared to the one produced by the parental strain (Ferreira et al., 2007). Therefore, it has been hypothesized that by promoting the formation of mature biofilms, the EPS may enhance bacterial survival in CF lung, which leads to the impossibility to efficiently eradicate $B c c$ infections.

\section{EMERGENCE OF BURKHOLDERIA MORPHOTYPE VARIATION DURING CHRONIC LUNG INFECTIONS}

The initial colonization of CF lungs by $P$. aeruginosa is made by environmental strains that during the course of infection 
developed the mucoid phenotype caused by production of alginate (Pedersen et al., 1992). To evaluate whether Burkholderia shows such variation during lung colonization, $560 \mathrm{Bcc}$ sequential isolates recovered from $100 \mathrm{CF}$ patients were screened for EPS production by analyzing their mucoid phenotype in EPSproducing medium (Zlosnik et al., 2008). Fifteen mucoid phenotypic transitions were observed: 13 from mucoid-to-non-mucoid were detected in B. multivorans, B. cenocepacia, and B. vietnamiensis; and two non-mucoid-to-mucoid transitions occurred in $B$. cenocepacia and B. vietnamiensis (Zlosnik et al., 2008). Further insights into the importance of this morphotypic variation in adaptation to lung environment came from two pairs of sequential isolates recovered from patients chronically infected for several years. The first pair of sequential isolates characterized was from B. cenocepacia and the isolates were recovered within 10 months of each other (Conway et al., 2004). These isolates showed genomic alterations as visible by their genome SpeI-restriction pattern, by mutations in the QS regulator encoding gene cepR and deletion of a region from the pathogenicity island present in the mucoid isolate (Zlosnik and Speert, 2010; McKeon et al., 2011). In addition, the mucoid isolate had decreased expression of virulence factors such as the nematocidal protein AidA or the zinc metalloprotease $\mathrm{ZmpA}$, was less resistant to oxidative stress, produced less biofilm, but showed a lower clearance rate from the lungs of $\mathrm{BALB} / \mathrm{c}$ mice when compared to the clonal non-mucoid isolate (Conway et al., 2004; Zlosnik and Speert, 2010). The second pair of clonal sequential isolates belongs to $B$. multivorans and the isolates recovered within a 6-month period. The mucoid isolate had decreased long-term survival rate in nutrient depleted medium, produced less biofilm, had increased expression of virulenceassociated genes, higher growth rate under microaerophilic conditions, increased motility, and was shown to be more virulent in Galleria mellonella (Silva et al., 2011). Since only two pairs of isolates with a mucoid vs. non-mucoid morphotype were evaluated it is early to take conclusions about the typical phenotypic profile of the non-mucoid isolates arising during chronic respiratory lung infections. Nevertheless, it is evident that the emergence of mucoid vs. non-mucoid morphotype variation in the lung of CF patients is correlated with metabolic changes, different survival ability under stress conditions, altered biofilm formation, motility, chemotaxis, and virulence (Figure 4).

The relationship of mucoid morphotype variation within adaptation to the lung environment and disease progression is not yet known. If EPS-producing bacteria could be favored to persist in the lungs by avoiding the immune system, it is also true that the non-EPS producers, by their ability to produce more biofilm and displaying higher survival rate under nutrient limitation, may be adapted as well to persist in the lungs. As regards the virulence potential of the mucoid and non-mucoid clonal isolates, the data obtained so far for mice and insects showed that the mucoid isolates were more virulent (Conway et al., 2004; Silva et al., 2011). Nevertheless, these data report to two isolates only and no ultimate conclusion about their virulence potential can be made. Besides, a study focused on the virulence potential of mucoid and non-mucoid clinical isolates in CF patient's lung function decline, suggested that patients infected with non-mucoid isolates had worst prognosis (Zlosnik et al., 2011).

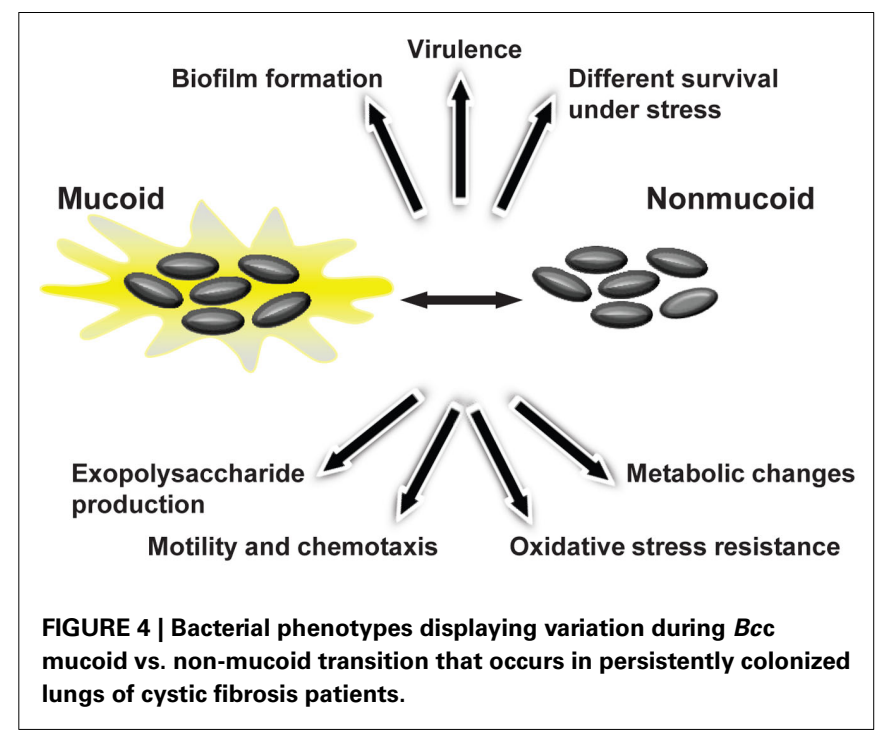

The cues triggering mucoid morphotype variation of $B c c$ during their persistence in the CF lung remain unknown. The only in vitro condition described so far that induces B. cenocepacia mucoid vs. non-mucoid transition is high doses of the antibiotics ceftazidime and ciprofloxacin (Zlosnik et al., 2011). As regards the molecular mechanisms behind the morphotype variation they are completely unidentified. During chronic lung infection genomic alterations including point mutations, deletion, and duplication of genes arise as it was found for the two pairs of $B c c$ isolates where the mucoid morphotype variation was characterized (Zlosnik and Speert, 2010; Silva et al., 2011). Whether these alterations are responsible for the observed phenotypes is unknown.

\section{CONCLUDING REMARKS}

The genus Burkholderia has the genetic capacity to produce several different secreted extracellular polysaccharides, although only cepacian biosynthetic mechanisms have been studied with some detail by the identification of the genes involved and characterization of some of the Bce proteins. However, no information exists regarding genetics and regulation of other polysaccharides identified in Burkholderia, some of which are often coexpressed with cepacian. The ability of Burkholderia to produce different exopolysaccharides raises questions on which environmental signals induce production of one polysaccharide vs. others and their specific roles to provide a survival advantage in different ecological niches such as soil, water, or during host/bacteria interactions. In particular, efforts have been made to understand the relevance of EPS production in virulence and persistence of $B c c$ bacteria in $\mathrm{CF}$ lung, but the role of EPS in Burkholderia virulence remains an open issue. While some evidences point out to EPS as a virulence factor, others indicate a major role in persistence. Furthermore, during the course of chronic lung infection genotypic and mucoid morphotype variation occurs, with clonal isolates displaying different phenotypes and virulence levels. This shows that EPS biosynthesis can be critical during Burkholderia lung infection, likely as part of a complex regulation network interfering with many other bacterial 
properties that change during infection and represent important bacterial adaptations to the CF lung. Further research is needed to understand the conditions that trigger such phenotypic conversions as well as the regulatory mechanisms underlying the cepacian biosynthesis.

\section{REFERENCES}

Bartholdson, S. J., Brown, A. R., Mewburn, B. R., Clarke, D. J., Fry, S. C., Campopiano, D. J., and Govan, J. R. (2008). Plant host and sugar alcohol induced exopolysaccharide biosynthesis in the Burkholderia cepacia complex. Microbiology 154, 2513-2521.

Benincasa, M., Mattiuzzo, M., Herasimenka, Y., Cescutti, P., Rizzo, R., and Gennaro, R. (2009). Activity of antimicrobial peptides in the presence of polysaccharides produced by pulmonary pathogens. J. Pept. Sci. 15, 595-600.

Bylund, J., Burgess, L.-A., Cescutti, P., Ernst, R. K., and Speert, D. P. (2006). Exopolysaccharides from Burkholderia cenocepacia inhibit neutrophil chemotaxis and scavenge reactive oxygen species. J. Biol. Chem. 281, 2526-2532.

Caraher, E., Reynolds, G., Murphy, P., Mcclean, S., and Callaghan, M. (2007). Comparison of antibiotic susceptibility of Burkholderia cepacia complex organisms when grown planktonically or as biofilm in vitro. Eur. J. Clin. Microbiol. Infect. Dis. 26, 213-216.

Cerantola, S., Lemassu-Jacquier, A., and Montrozier, H. (1999). Structural elucidation of a novel exopolysaccharide produced by a mucoid clinical isolate of Burkholderia cepacia. Characterization of a trisubstituted glucuronic acid residue in a heptasaccharide repeating unit. Eur. J. Biochem. 260, 373-383.

Cerantola, S., Marty, N., and Montrozier, H. (1996). Structural studies of the acidic exopolysaccharide produced by a mucoid strain of Burkholderia cepacia, isolated from cystic fibrosis. Carbohydr. Res. 285, 59-67.

Cescutti, P., Bosco, M., Picotti, F., Impallomeni, G., Leitao, J. H., Richau, J. A., and Sa-Correia, I. (2000). Structural study of the exopolysaccharide produced by a clinical isolate of Burkholderia cepacia. Biochem. Biophys. Res. Commun. 273, 1088-1094.

Cescutti, P., Foschiatti, M., Furlanis, L., Lagatolla, C., and Rizzo, R. (2010). Isolation and characterisation of the biological repeating unit of cepacian, the exopolysaccharide produced by bacteria of the Burkholderia cepacia complex. Carbohydr. Res. 345, 1455-1460.

Cescutti, P., Impallomeni, G., Garozzo, D., Sturiale, L., Herasimenka, Y., Lagatolla, C., and Rizzo, R. (2003). Exopolysaccharides produced by a clinical strain of Burkholderia cepacia isolated from a cystic fibrosis patient. Carbohydr. Res. 338, 2687-2695.

Collins, R. F., Beis, K., Clarke, B. R., Ford, R. C., Hulley, M., Naismith, J. H., and Whitfield, C. (2006). Periplasmic protein-protein contacts in the inner membrane protein Wzc form a tetrameric complex required for the assembly of Escherichia coli 2144-2150.

Conway, B.-A. D., Chu, K. K., Bylund, J., Altman, E., and Speert, D. P. (2004). Production of exopolysaccharide by Burkholderia cenocepacia results in altered cell-surface interactions and altered bacterial clearance in mice. J. Infect. Dis. 190, 957-966.

Coutinho, P. M., Deleury, E., Davies, G. J., and Henrissat, B. (2003). An evolving hierarchical family classification for glycosyltransferases. $J$. Mol. Biol. 328, 307-317.

Denny, T. P. (1995). Involvement of bacterial polysaccharides in plant pathogenesis. Annu. Rev. Phytopathol. 33, 173-197.

Devescovi, G., and Venturi, V. (2006). The Burkholderia cepacia rpoE gene is not involved in exopolysaccharide production and onion pathogenicity. Can. J. Microbiol. 52, 260-265.

Dong, C., Beis, K., Nesper, J., BrunkanLamontagne, A. L., Clarke, B. R., Whitfield, C., and Naismith, J. H. (2006). Wza the translocon for E. coli capsular polysaccharides defines a new class of membrane protein. Nature 444, 226-229.

Ferreira, A. S., Leitão, J. H., Silva, I. N., Pinheiro, P. F., Sousa, S. A., Ramos, C. G., and Moreira, L. M. (2010). Distribution of cepacian biosynthesis genes among environmental and clinical Burkholderia strains and role of cepacian exopolysaccharide in resistance to stress conditions. Appl. Environ. Microbiol. 76, 441-450. group 1 capsules. J. Biol. Chem. 281,

\section{ACKNOWLEDGMENTS}

This work was supported by FEDER and Fundação para a Ciência e a Tecnologia, Portugal (contracts PTDC/QUI-BIQ/118260/2010 and PTDC/BIA-MIC/113733/2009, a Post-doctoral grant to Ana S. Ferreira and a doctoral grant to Inês N. Silva).

Ferreira, A. S., Leitão, J. H., Sousa, S. A., Cosme, A. M., Sá-Correia, I. and Moreira, L. M. (2007). Functional analysis of Burkholderia cepacia genes $b c e D$ and $b c e F$, encoding a phosphotyrosine phosphatase and a tyrosine autokinase, respectively: role in exopolysaccharide biosynthesis and biofilm formation. Appl. Environ. Microbiol. 73, 524-534.

Fraysse, N., Couderc, F., and Poinsot, V. (2003). Surface polysaccharide involvement in establishing the Rhizobium-legume symbiosis. Eur. J. Biochem. 270, 1365-1380.

Gonzalez, J. E., York, G. M., and Walker, G. C. (1996). Rhizobium meliloti exopolysaccharides: synthesis and symbiotic function. Gene 179, 141-146.

Govan, J. R., Brown, P. H., Maddison, J., Doherty, C. J., Nelson, J. W., Dodd, M., Greening, A. P., and Webb, A. K. (1993). Evidence for transmission of Pseudomonas cepacia by social contact in cystic fibrosis. Lancet 342, 15-19.

Hallack, L. F., Passos, D. S., Mattos, K. A. Agrellos, O. A., Jones, C., MendoncaPreviato, L., Previato, J. O., and Todeschini, A. R. (2010). Structural elucidation of the repeat unit in highly branched acidic exopolysaccharides produced by nitrogen fixing Burkholderia. Glycobiology 20, 338-347.

Hentzer, M., Teitzel, G. M., Balzer, G. J., Heydorn, A., Molin, S., Givskov, M., and Parsek, M. R. (2001). Alginate overproduction affects Pseudomonas aeruginosa biofilm structure and function. J. Bacteriol. 183, 5395-5401.

Hoiby, N., Ciofu, O., and Bjarnsholt, T. (2010). Pseudomonas aeruginosa biofilms in cystic fibrosis. Future Microbiol. 5, 1663-1674

Lacour, S., Bechet, E., Cozzone, A. J., Mijakovic, I., and Grangeasse, C. (2008). Tyrosine phosphorylation of the UDP-glucose dehydrogenase of Escherichia coli is at the crossroads of colanic acid synthesis and polymyxin resistance. PLoS ONE 3, e3053. doi:10.1371/journal.pone. 0003053

Lebeer, S., Claes, I. J., Verhoeven, T. L., Vanderleyden, J., and De Keersmaecker, S. C. (2011). Exopolysaccharides of Lactobacillus rhamnosus GG form a protective shield against innate immune factors in the intestine. Microb. Biotechnol. 4, 368-374.

Loutet, S. A., Bartholdson, S. J., Govan, J. R., Campopiano, D. J., and Valvano, M. A. (2009). Contributions of two UDP-glucose dehydrogenases to viability and polymyxin $B$ resistance of Burkholderia cenocepacia. Microbiology 155, 2029-2039.

Mattos, K. A., Jones, C., Heise, N., Previato, J. O., and MendoncaPreviato, L. (2001). Structure of an acidic exopolysaccharide produced by the diazotrophic endophytic bacterium Burkholderia brasiliensis. Eur. J. Biochem. 268, 3174-3179.

McKeon, S. A., Nguyen, D. T., Viteri, D. F., Zlosnik, J. E., and Sokol, P. A. (2011). Functional quorum sensing systems are maintained during chronic Burkholderia cepacia complex infections in patients with cystic fibrosis. J. Infect. Dis. 203, 383-392.

Minic, Z., Marie, C., Delorme, C., Faurie, J. M., Mercier, G., Ehrlich, D., and Renault, P. (2007). Control of EpsE, the phosphoglycosyltransferase initiating exopolysaccharide synthesis in Streptococcus thermophilus, by EpsD tyrosine kinase. J. Bacteriol. 189, 1351-1357.

Moreira, L. M., Videira, P. A., Sousa, S. A., Leitão, J. H., Cunha, M. V., and Sá-Correia, I. (2003). Identification and physical organization of the gene cluster involved in the biosynthesis of Burkholderia cepacia complex exopolysaccharide. Biochem. Biophys. Res. Commun. 312, 323-333.

Nielsen, L., Li, X., and Halverson, L. J. (2011). Cell-cell and cellsurface interactions mediated by cellulose and a novel exopolysaccharide contribute to Pseudomonas putida biofilm formation and fitness under water-limiting conditions. Environ. Microbiol. 13, 1342-1356.

Nimtz, M., Wray, V., Domke, T., Brenneke, B., Haussler, S., and Steinmetz, I. (1997). Structure of an acidic exopolysaccharide of Burkholderia pseudomallei. Eur. J. Biochem. 250, 608-616.

Pedersen, S. S., Hoiby, N., Espersen, F., and Koch, C. (1992). Role of alginate in infection with mucoid Pseudomonas aeruginosa in cystic fibrosis. Thorax 47, 6-13. 
Potts, M. (1994). Desiccation tolerance of prokaryotes. Microbiol. Rev. 58, 755-805.

Ramsey, D. M., and Wozniak, D. J. (2005). Understanding the control of Pseudomonas aeruginosa alginate synthesis and the prospects for management of chronic infections in cystic fibrosis. Mol. Microbiol. 56, 309-322.

Richau, J. A., Leitão, J. H., Correia, M., Lito, L., Salgado, M. J., Barreto, C., Cescutti, P., and SáCorreia, I. (2000a). Molecular typing and exopolysaccharide biosynthesis of Burkholderia cepacia isolates from a Portuguese cystic fibrosis center. J. Clin. Microbiol. 38, 1651-1655.

Richau, J. A., Leitão, J. H., and SáCorreia, I. (2000b). Enzymes leading to the nucleotide sugar precursors for exopolysaccharide synthesis in Burkholderia cepacia. Biochem. Biophys. Res. Commun. 276, 71-76.

Rocha, J., Popescu, A. O., Borges, P., Mil-Homens, D., Moreira, L. M., SáCorreia, I., Fialho, A. M., and Frazão, C. (2011). Structure of Burkholderia cepacia UDP-glucose dehydrogenase (UGD) BceC and role of Tyr10 in final hydrolysis of UGD thioester intermediate. J. Bacteriol. 193, 3978-3987.

Schwab, U., Leigh, M., Ribeiro, C., Yankaskas, J., Burns, K., Gilligan, P., Sokol, P., and Boucher, R. (2002). Patterns of epithelial cell invasion by different species of the Burkholderia cepacia complex in well-differentiated human airway epithelia. Infect. Immun. 70, 4547-4555.

Silva, I. N., Ferreira, A. S., Becker, J. D., Zlosnik, J. E., Speert, D. P., He, J., Mil-Homens, D., and Moreira, L. M. (2011). Mucoid morphotype variation of Burkholderia multivorans during chronic cystic fibrosis lung infection is correlated with changes in metabolism, motility, biofilm formation and virulence. Microbiology 157, 3124-3137.

Sokol, P. A., Sajjan, U., Visser, M. B., Gingues, S., Forstner, J., and Kooi, C. (2003). The CepIR quorum-sensing system contributes to the virulence of Burkholderia cenocepacia respiratory infections. Microbiology 149, 3649-3658.

Sousa, S. A., Moreira, L. M., and Leitao, J. H. (2008). Functional analysis of the Burkholderia cenocepacia J2315 BceA J protein with phosphomannose isomerase and GDP-Dmannose pyrophosphorylase activities. Appl. Microbiol. Biotechnol. 80, 1015-1022.

Sousa, S. A., Moreira, L. M., Wopperer, J. Eberl, L., Sá-Correia, I., and Leitão, J. H. (2007a). The Burkholderia cepacia bceA gene encodes a protein with phosphomannose isomerase and GDP-D-mannose pyrophosphorylase activities. Biochem. Biophys. Res. Commun. 353, 200-206.

Sousa, S. A., Ulrich, M., Bragonzi, A., Burke, M., Worlitzsch, D., Leitão, J. H., Meisner, C., Eberl, L., Sá-Correia, I., and Doring, G. (2007b). Virulence of Burkholderia cepacia complex strains in gp9lphox-/- mice. Cell. Microbiol. 9, 2817-2825.

Sousa, S. A., Ramos, C. G., Moreira, L. M., and Leitão, J. H. (2010). The $h f q$ gene is required for stress resistance and full virulence of Burkholderia cepacia to the nematode Caenorhabditis elegans. Microbiology 156, 896-908.

Suarez-Moreno, Z. R., Devescovi, G., Myers, M., Hallack, L., MendoncaPreviato, L., Caballero-Mellado, J., and Venturi, V. (2010). Commonalities and differences in regulation of $\mathrm{N}$-acyl homoserine lactone quorum sensing in the beneficial plant-associated Burkholderia species cluster. Appl. Environ. Microbiol. 76, 4302-4317.

Tocilj, A., Munger, C., Proteau, A., Morona, R., Purins, L., Ajamian, E. Wagner, J., Papadopoulos, M., Van Den Bosch, L., Rubinstein, J. L., Fethiere, J., Matte, A., and Cygler, M. (2008). Bacterial polysaccharide co-polymerases share a common framework for control of polymer length. Nat. Struct. Mol. Biol. 15, 130-138.

Vanhaverbeke, C., Heyraud, A. Achouak, W., and Heulin, T. (2001). Structural analysis of the exopolysaccharide from Burkholderia caribensis strain MWAP71. Carbohydr. Res. 334, 127-133.

Vanhaverbeke, C., Heyraud, A., and Mazeau, K. (2003). Conformational analysis of the exopolysaccharide from Burkholderia caribensis strain MWAP71: impact on the interaction with soils. Biopolymers 69, 480-497.

Videira, P. A., Garcia, A. P., and SáCorreia, I. (2005). Functional and topological analysis of the Burkholderia cenocepacia priming glucosyltransferase BceB, involved in the biosynthesis of the cepacian exopolysaccharide. J. Bacteriol. 187, 5013-5018.

Vincent, C., Duclos, B., Grangeasse, C., Vaganay, E., Riberty, M., Cozzone, A. J., and Doublet, P. (2000). Relationship between exopolysaccharide production and proteintyrosine phosphorylation in gramnegative bacteria. J. Mol. Biol. 304 311-321.

Whitfield, C. (2006). Biosynthesis and assembly of capsular polysaccharides in Escherichia coli. Annu. Rev. Biochem. 75, 39-68.

Wugeditsch, T., Paiment, A., Hocking, J., Drummelsmith, J., Forrester, C., and Whitfield, C. (2001). Phosphorylation of Wzc, a tyrosine autokinase, is essential for assembly of group 1 capsular polysaccharides in
Escherichia coli. J. Biol. Chem. 276, 2361-2371.

Zlosnik, J. E., Costa, P. S., Brant, R., Mori, P. Y., Hird, T. J., Fraenkel, M. C., Wilcox, P. G., Davidson, A. G., and Speert, D. P. (2011). Mucoid and nonmucoid Burkholderia cepacia complex bacteria in cystic fibrosis infections. Am. J. Respir. Crit. Care Med. 183, 67-72.

Zlosnik, J. E., Hird, T. J., Fraenkel, M. C., Moreira, L. M., Henry, D. A., and Speert, D. P. (2008). Differential mucoid exopolysaccharide production by members of the Burkholderia cepacia complex. J. Clin. Microbiol. 46, 1470-1473.

Zlosnik, J. E., and Speert, D. P. (2010). The role of mucoidy in virulence of bacteria from the Burkholderia cepacia complex: a systematic proteomic and transcriptomic analysis. J. Infect. Dis. 202, 770-781.

Conflict of Interest Statement: The authors declare that the research was conducted in the absence of any commercial or financial relationships that could be construed as a potential conflict of interest.

Received: 26 August 2011; accepted: 21 November 2011; published online: 15 December 2011.

Citation: Ferreira AS, Silva IN, Oliveira VH, Cunha $R$ and Moreira LM (2011) Insights into the role of extracellular polysaccharides in Burkholderia adaptation to different environments. Front. Cell. Inf. Microbio. 1:16. doi: 10.3389/fcimb.2011.00016

Copyright (C) 2011 Ferreira, Silva, Oliveira, Cunha and Moreira. This is an open-access article distributed under the terms of the Creative Commons Attribution Non Commercial License, which permits non-commercial use, distribution, and reproduction in other forums, provided the original authors and source are credited. 